\title{
Mutual Feedback Between Star Formation and Nuclear Activity
}

\author{
Gian Luigi Granato ${ }^{1,2}$ \\ ${ }^{1}$ INAF-OApd, Vicolo Osservatorio 5, Padova, Italy \\ ${ }^{2}$ SISSA, Via Beirut 4, Trieste, Italy \\ email: gianluigi.granato@oapd.inaf.it
}

\begin{abstract}
In this invited contribution I review the justifications for the attempts, currently very popular, to include in semi-analytic models of galaxy formation prescriptions to describe the mutual link between the star formation and nuclear activity in galaxies, which has been for surprisingly long time neglected.
\end{abstract}

Keywords. galaxies: formation; galaxies: active

\section{Introduction}

Since a few years ago, studies of galaxy formation have been affected by uncertainties both in the cosmology and in the most relevant physical processes. But now we are in the so called precision cosmology era, which implies that the background model is relatively well defined, and we can compute with reasonable confidence and precision the evolution of the dynamically dominant dark matter (DM) component, ruled essentially only by gravity. By converse the main physical processes driving galaxy formation, in particular the evolution of baryonic (ordinary) matter, which allow us to see galaxies, are extremely complex and still hotly debated.

Indeed, to compare scenarios and ideas of galaxy formation with observations there are two strong sources of difficulties and uncertainties.

- The first is to predict the evolution of ordinary matter, which is highly non linear, and above all is driven mostly by processes occurring well below the resolution of any feasible simulation. These precesses are usually referred to as sub-grid physics, include star formation (SF), accretion onto super-massive black holes (SMBH), merging of $\mathrm{BH}$, and are also poorly understood from a physical point of view.

- Another point that has been for long time under-appreciated is the modelling of the interaction of photons produced by stars and accretion processes with the dusty ISM, likely more and more important at higher and higher $\mathrm{z}$, to the point that it is likely that a major fraction of SF activity at high $\mathrm{z}$ is completely hidden to optical searches.

In this contribution I will discuss a few aspects of the first point, though our group has devoted since long a lot of effort also to cope the second problem, developing what is still the most advanced and flexible tool to predict the spectral energy distribution of galaxies in the presence of dust (GRASIL, Silva et al. 1998; Vega et al. 2005).

\section{The myth of first principles models}

Due to the formidable task of computing the evolution of baryons within galaxies in cosmological context, it is fair to say that first principles or ab-initio models do not exist, despite some optimistic claims. Indeed in any computation sub-grid physics is treated (if ever) through semi-analytic-like recipes, that is formulae containing many free 
parameters, which try to describe sub-grid physics by means of relationships between integrated quantities. Unfortunately, the model outcomes are heavily affected, if not driven, by these formulae.

An illuminating example, related to the topic of this talk, are the simulations of merging between disk galaxies carried by Di Matteo et al. (2005). They included a rough sub-grid treatment of the induced accretion onto a central SMBH and of the feed-back that its activity may have on the evolution of the system. Moreover, these are not simulations of cosmological volumes, thus in principle sub-grid phenomena should be less a problem. However, the striking result is that two comparison simulations, carried respectively with and without the inclusion of these effects, lead to completely different results in terms of star formation histories and final fate of the initial gas. Without AGN the gas is almost completely turned into stars, while with AGN feed-back only about $50 \%$ goes into stars and the rest is expelled from the halo. Thus an ingredient which has to be treated with crude and uncertain approximations dominates the predicted evolution. This casts some perplexity on the rationale to attempt fully numerical simulations of galaxy formation in cosmological volumes, and is the basic justification of the wealth of semi-analytic models on the market, in which all the processes involving baryons are treated by means of simplified "recipes" or "prescriprions".

\section{Standard SAMs, their successes and their failures}

Indeed, the most extensive comparisons between different scenarios and the rich data sets now available are done by means of fully semi-analytic models (SAM) for baryons, possibly in the form of post-processing of gravity-only simulations for DM. By definition, these models are based on many a-priori assumptions on the general development of galaxies. More proper naming could be toy models.

Almost all SAMs adopt a-priori what we can call a disk galaxy merger driven sequence of processes leading to galaxy populations at any redshift and in particular locally. This is based mainly to two assumptions: (i) that the first outcome of gas cooling in DMH are disks of gas supported by rotation (e.g. Rees \& Ostriker 1977, White \& Rees 1978), and in which there is only a mild SF activity, while (ii) the only cause of violent episodes of SF at any redshift are mergers between these disks, which are also the main path to form spheroidal galaxies (e.g. White \& Frank 1991, Cole et al. 2000).

These assumptions may be not completely correct. Indeed, despite the high number of adjustable parameters involved, it has become increasingly clear over the years that calculations based on this general scheme, besides remarkable successes in reproducing several properties of galaxy population, show severe mismatches with some very basic observations. Namely: (i) without ad-hoc and physically unjustified assumptions, it is impossible to reproduce the sharp break at the bright end of the Local Luminosity Function, in particular now that the low baryon fraction used in old models has been ruled out by observations: models predict too many (and too young-blue) bright galaxies (e.g. Benson et al 2003); (ii) by converse, beyond $z \sim 1-1.5$, most models tend to predict too few bright galaxies, compared with the wealth of determinations done in the past few years; (iii) these are aspects of cosmic downsizing: star formation and accretion onto SMBH decline more with cosmic time for larger system than for smaller ones, a fact which is at odd with naive expectations of hierarchical growth; (iv) the observed absence of cooling flows in the centers of real rich clusters, where cooling times are much shorter than the Hubble time; (v) the properties of local E galaxies, such as the colors or the alpha enhancement as a function of mass are not well reproduced. Most people think 
that these difficulties may well be all facets of the same problem, suggesting that some key ingredient is missing or/and the entire scheme needs a substantial revision.

\section{Possible solution from joint evolution of QSO and Spheroids}

It is at present very popular the idea that at least part of the solution could come from an ingredient that only in the very last few years started to be taken into account in some models, i.e. the mutual influence or feed-back (FB) of star formation in galaxies and the development of SMBH-QSO at their centers. This influence is hinted by several empirical and also theoretical facts, for instance the well established local correlation between the mass of the central SMBH and several properties of the hosting spheroid (the luminosity, the mass, the velocity dispersion), the similarity of the cosmic development of $\operatorname{SFR}(\mathrm{z})$ and the luminosity of QSO per unit volume, or the fact that simulations of merging between galaxies drive flows of gas toward the central regions, creating an environment at least favorable to promote SMBH accretion.

Having now a relatively good determination of the almost constant ratio of stellar mass in spheroids and the mass of the hosted SMBH $\sim 1000$, it is interesting to compare the binding energy of spheroids with the energy released by the SMBH to accrete this mass. For a typical $L^{*}$ galaxy, the latter turns out to be more than two orders of magnitude greater than the former, which means that a quite small coupling between the energy released by the AGN and the ISM is sufficient to have a significant effect. It is interesting to compare also the binding energy with the energy released by SNae during the assembly of the same spheroid, which is only about a factor 10 greater than the former.

Then the energy available is large enough, though it is not clear if and how a (small) fraction of this energy can be transferred to the ISM. Several possibilities have been studied, in particular: radiation pressure, mostly on dust grains: a dusty medium is orders of magnitude more effective in absorbing momentum than a dust-free one; Compton or line radiative heating; kinetic outflows from AGN, such as those observed in jet and BAL (likely generated by radiation pressure on resonant lines relatively close to the central engine). Another proposed possibility is that the AGN may act as a kind of catalyst to enhance the effectiveness of SNae feed-back (Monaco 2004).

Only in the very last few years these effects started to be explicitly considered in SAMs. This has been done along two quite distinct lines that should not be confused.

- Granato et al. 2004, Monaco \& Fontanot 2005; Menci et al. 2006 considered the FB associated with the main phase of the BH growth, related to the bright high-z QSOs, as a way to sterilize massive high-z galaxies, which instead are little affected by SNae FB, due to the depth of their potential well.

- More recently, and in the context of more standard SAMs, it has been considered (only) FB associated with lower redshift, low accretion rate phase of AGN, in which almost all the accretion energy is used to halt cooling flows and avoid overproduction of local bright galaxies (Bower et al. 2006, Croton et al. 2006)

In general in this second set of works little attempt, or none at all, has been done in treating the build up of SMBH and the physical nature of the feedback.

\section{The ABC scenario}

The first SAM in which a key role has been invoked for the reciprocal feed-back between star formation and AGN activity is the "Antihierarchical Baryonic Collapse" (ABC) proposed by Granato et al. 2004 (see also Granato et al. 2001 for a more phenomenological treatment). This model, which is fully embedded in the $\Lambda$ CDM hierarchical growth of 
DM halos and is focused on the formation of spheroids, adopts prescriptions to describe the baryonic physics which reverse the order with which spheroidal galaxies and high-z QSOs complete their formation, as indicated by the various evidences of downsizing. This is obtained by a combination of two ingredients: (i) revised prescriptions for the $\mathrm{SF}$ in massive high redshift galactic halos $\left(M_{v i r} \gtrsim 10^{12} M_{\odot}\right)$, which allows SFR as high as thousands of solar masses per year, as implied by observations of sub-mm galaxies, and (ii) the inclusion of a treatment of the growth by accretion of a SMBH promoted by this huge SF activity (positive feed-back between SF and accretion), which at some point becomes so powerful to clean the ISM and quench any further SF and accretion (negative QSO feed-back).

The ABC scenario predicts a well defined evolutionary sequence leading to local ellipticals with dormant SMBH. The sequence begins with a high redshift phase of huge, dust enshrouded SF activity best detectable in the submm spectral region, and lasting about 0.5 Gyr. This phase is ended by the strong feed-back generated by the QSO phase, due to the growth of a $\mathrm{SMBH}$, which is promoted by the huge star formation activity during the previous SMG phase. This feedback sterilizes the system, which then evolve almost passively, thus it predicts a sizeable population of massive and dead galaxies already at high-z. The model leads (in one shot) to predictions in general agreement with many observations which are at least disturbing for traditional SAMs.

For the description of the model and how it compares with observations of these populations of objects the reader is referred to Granato et al. (2001, 2004), Silva et al. (2005); Granato et al. (2006) Lapi et al. (2006); Silva et al. in preparation.

\section{Acknowledgements}

The ideas presented in this invited contribution result from a long standing collaboration with several colleagues, in particular, listed in reverse alphabetic order, L. Silva, F. Shankar, A. Lapi, G. De Zotti, L. Danese and A. Bressan. Work in part supported by EC under CONTRACT MRTN-CT-2004-503929.

\section{References}

Benson, A. J., Bower, R. G., Frenk, C. S., Lacey, C. G., Baugh, C. M., \& Cole, S., 2003, ApJ, $599,38$.

Bower, R. G., Benson, A. J., Malbon, R., Helly, J. C., Frenk, C. S., Baugh, C. M., Cole, S., \& Lacey, C. G., 2006, MNRAS, 370, 645.

Cole, S., Lacey, C. G., Baugh, C. M., \& Frenk, C. S., 2000, MNRAS, 319, 168.

Croton, D. J., et al., 2006, MNRAS, 365, 11.

Di Matteo, T., Springel, V., \& Hernquist, L., 2005, Natur, 433, 604.

Granato, G. L., Silva, L., Monaco, P., Panuzzo, P., Salucci, P., De Zotti, G., \& Danese L., 2001, MNRAS, 324, 757.

Granato, G. L., De Zotti, G., Silva, L., Bressan, A., \& Danese, L., 2004, ApJ, 600, 580, (GDS04).

Lapi, A., Shankar, F., Mao, J., Granato, G. L., Silva, L., De Zotti, G., \& Danese, L., 2006, ApJ, $650,42$.

Menci, N., Fontana, A., Giallongo, E., Grazian, A., \& Salimbeni, S., 2006, ApJ, 647, 753.

Monaco, P., \& Fontanot, F., 2005, MNRAS, 359, 283.

Rees, M. J., \& Ostriker, J. P., 1977, MNRAS, 179, 541.

Silva, L., Granato, G. L., Bressan, A., \& Danese, L., 1998, ApJ, 509, 103.

Silva, L., De Zotti, G., Granato, G. L., Maiolino, R., \& Danese, L., 2005, MNRAS, 357, 1295.

Vega, O., Silva, L., Panuzzo, P., Bressan, A., Granato, G. L., \& Chavez, M., 2005, MNRAS, 364, 1286.

White, S. D. M., \& Rees, M. J., 1978, MNRAS, 183, 341.

White, S. D. M., \& Frenk, C. S., 1991, ApJ, 379, 52. 


\section{Discussion}

Mike Hudson: How does the downsizing seen in red galaxies at low redshift arises in your model?

Gian Luigi Granato: Black hole growth is more efficient in high mass haloes and this then cuts off star formation in these systems at an earlier time.

Claudia Maraston: Do your models require a late $(\mathrm{z}<1)$ mass assembly of massive early-type galaxies, like the models by de Lucia et al.

Gian Luigi Granato: No, in this model, not only the formation of stars but also their assembly into a single galaxy occurs at high $\mathrm{z}(>2)$. 